\title{
An Integrated Model for Simulating Activities and Optimizing Space Function in Educational Facility
}

\author{
Ren-Jye Dzeng, Chin-Teng Lin, and Fan-Yi Hsiao
}

\begin{abstract}
Determining the function for each space in a building plays an important role for assessing the service level of an educational facility. The function layout affects how users move between spaces during their participation of activities in the facility. This research describes the integration of an activity-based simulation model and a space-function assignment optimization model with six phases, including data collection, data preparation, data mining, optimization, verification, and refinement. The paper also uses a real case to demonstrate the use of the proposed model, and has shown a 4.96\% improvement on the objective value compared to the contracted architect's original plan.
\end{abstract}

Index Terms-Function space assignment, optimization, activity simulation, movement simulation.

\section{INTRODUCTION}

The layout and function assignment of space, along with other factors such as physical environment and energy use, is one important factor in determining the service level of a building. Most research in the evaluation of building service during the design phase focuses on the simulation or calculation of physical environment of a building such as lighting, temperature, humidity [1], [2], or energy usage [3]. Currently, evaluating how the space layout and function assignment fit the prospective users' activities are still difficult in the design phase because of lacking an appropriate tool capable of modeling the users' activities and simulating the activities in the designed layout.

There are three variables involved that may affect the service level, namely space function assignment, the activities taking place in the spaces, and the way in which users move between the spaces. Function assignment related research have been widely applied to many different real situations, such as the planning of buildings in university campuses, arrangement of departments in hospitals, warehouse management and distribution strategies, minimization of the total wire length in electronic circuits, ordering of correlated data in magnetic tapes and others [4]. However, there is very limited study on the simulation of the users' activities and movements in the literature of function-space assignment.

Hoes [5] stated that the behavior of user activities is one of the important causes influencing the result of buildings

Manuscript received April 10, 2013; revise June 20, 2013.

R. J. Dzeng and F. Y. Hsiao are with the Dept. of Civil Engr., National Chiao-Tung University, 1001, University Road, Hsin-Chu, Taiwan (e-mail: rjdzeng@mail.nctu.edu.tw, minlove.xiao@gmail.com).

C. T. Lin is with the Dept. of Electrical Engineering and Computer Science, National Chiao-Tung University, 1001, University Road, Hsin-Chu, Taiwan (e-mail: ctlin@mail.nctu.edu.tw). performance assessment based on buildings play a role of accommodating users' organizations and equipment, and enable their activities. The relation between the users and the building is mediated through the functional spaces where the activities take place [6]. Therefore, the level of service of indoor space in a building depends on whether the design of functional spaces assignments, corridors and stairs are satisfied the activities needs of each user. Recently, several researchers started to evaluate the service level by simulating and predicting user's activities in a building based on user activity models. Tabak et al. [7] proposed a prediction model for the frequency of intermediate activities during an office working day based on the probabilistic and S-curve methods. Shen et al. [8] combined user activity simulation with building information models to develop a pre-occupancy evaluation system to facilitate the designer-user communications. In order to find the best compromise between energy savings and comfort, Zimmermann et al. [9] developed a user activity model for the lighting control, heating and cooling system based on the multi-agent simulations. Lertlakkhanakul et al. [10] built a simulation platform for spatial interaction management in smart home using virtual reality technology which can be used to simulate smart home service configuration.

Research that tackles each individual variable helps the design in some ways. For example, simulation of activities alone helps understanding the space usage, and simulation of movement alone helps estimating traffic flow density and identifying corridor bottlenecks. However, tackling one variable without considering others may also leave out some important design information. For example, assigning functions to spaces without considering activities ignore the correlations among functions and may assign two highly correlated functions to two spaces far apart. Assigning functions to space without considering movement may ignore the total movement distances for participating activities, and the interference to the quiet spaces while users move from one function to another.

A few researchers started to consider multiple variables. Lee et al. [11] considered both function assignment and movement simulation by integrating ant colony optimization and continuous movement simulation to improve corridor layouts for a hospital building. Similarly, Dzeng et al. [12] integrated fast messy genetic algorithm (fmGA) optimization and discrete movement simulation to develop a FAMOS model (Function-space Assignment and Movement Simulation) for an educational building. However, none of researchers have integrated all three variables.

This study proposes a simulation-based assessment model that extends the FAMOS model by integrating the activity simulation. We first established the activity scheduling data 
for the users of a building using probability distributions based on the associated users' use. By using FAMOS, the function-space assignment was optimized and assessed using movement simulation. Finally, the proposed model took the movement distance, space occupancy per user per unit time, and flow density of corridors for the quantitative indexes to analyze the level of service of the building. The integration of activity simulation, space layout optimization, and movement simulation has the advantage of allowing the designer to assess the service level of space during the design phase at much more detailed level objectively with a guided optimized suggestion.

\section{ReSEARCh PROBlem AND PROPOSED Model}

Given a list of the spaces (e.g., large classroom, small classroom, administration office, meeting room) of an educational facility, and the activities (e.g., attending "Construction Methods" class, reading journals in the department library) in which types of users (e.g., sophomore, senior) may participate. Each space at each time slot can only be assigned with one activity. Given a known layout of the spaces, the research problem is to arrange functions for the spaces so that maximum service level can be achieved.

Fig. 1 shows the six phases of improving the function space assignment and its adopting problem-solving methods in this study, described as follows.

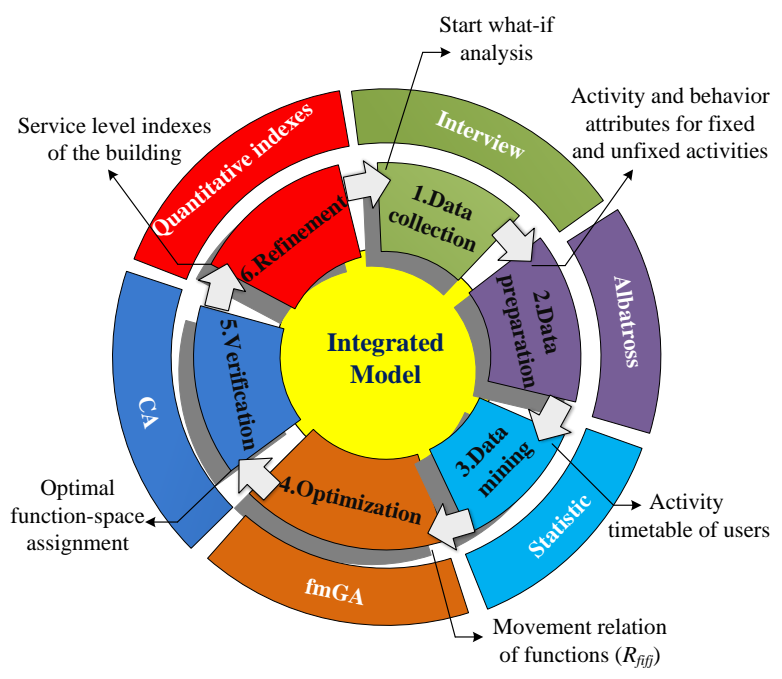

Fig. 1. Magnetization as a function of applied field.

\section{A. Phase 1 Data Collection}

The first step is to collect data, including fixed and unfixed activities and user groups. Users in an educational facility may share similarity in the activities they participate in. For example, the sophomore male students can be defined as a group. Members of a group may be associated with the same set of activities and their activity participating behaviors are similar with probabilistic deviation.

A group has attributes group-name (e.g., Junior students), group-ID (e.g., G001), number-of-members (e.g., 95), activity-participating-list (e.g., [(Act ID, behavior attributes)+]). The number-of-members determines the number of virtual users generated during the simulation. The activity-participating-list is a list of what activity and how the group member participates in. Each entry is an activity with a list of behavior attributes.

Doherty and Axhausen [13] stated that users tend to schedule their activities in a priority base, rather than time sequential way. In other words, the high priority activities are scheduled first, and the low priority activities are scheduled later when additional time slots are available. Based on the argument, this research defines two types of activities, i.e., the fixed and unfixed activities. The fixed activity is an activity whose schedule is predetermined and fixed weekly. Examples are attending a requisite or elective class, or a seminar. The unfixed activities include flexible activity and ad-hoc activity. Meeting with classmates for team assignment discussion is a flexible activity because an appointment needs to be made but the meeting times may be different each week. The ad-hoc activity is an activity that users carry out on their own accord without particular planning. Examples of ad-hoc activities are reading magazines in the department library, or getting packages at the department office.

Table I shows examples of fixed activity and unfixed activity. For example, the members of a group may participate in a fixed activity "Construction Methods" class starting at 11:10AM Thursday, 13:20PM Friday, and 14:20PM Friday, respectively. Each class lasts for 50 minutes at space F002 once a week for the period from Feb. 18th, 2013 to Jun. 21th, 2013. The average attendance rate of the group for this activity is 0.90 . The arrival time of attending the Friday class and the first Friday class for the group is a normal distribution with mean equal to 6.0 minutes $(-12 \% \times$ $50=6.0)$ ahead of class start time, and standard deviation of 3.0 minutes $(-6 \% \times 50=3.0)$. The arrival time of attending the second Friday class is a normal distribution with mean equal to 3.0 minutes $(-6 \% \times 50=3.0)$ ahead of class start time, and standard deviation of 1 minutes $(-2 \% \times 50=1)$. The specification shows that students are more punctual for the second class in consecutive classes.

TABLE I: EXAMPLES OF FIXED ACTIVITY AND UNFIXED ACTIVITY

\begin{tabular}{|c|c|c|c|c|c|}
\hline & \multirow{3}{*}{ Activity Example } & \multicolumn{2}{|c|}{ Fixed Activity } & \multicolumn{2}{|c|}{ Unfixed Activity } \\
\hline & & ID & Name & ID & Name \\
\hline & & F002 & $\begin{array}{l}\text { Construction } \\
\text { Methods }\end{array}$ & U002 & $\begin{array}{l}\text { Getting } \\
\text { packages }\end{array}$ \\
\hline \multirow{4}{*}{$\begin{array}{c}\text { Activity } \\
\text { Attributes }\end{array}$} & schedule-specs & \multicolumn{2}{|c|}{$\begin{array}{l}\text { [THU, 11:10,50,1] } \\
{[\text { FRI, 13:20,50,1] }} \\
{[\text { FRI, 14:20,50,1] }}\end{array}$} & \multicolumn{2}{|c|}{$\begin{array}{l}\text { [(TUE, WED, } \\
\text { THU) }(14: 10 \text {, } \\
15: 20)(3,5) \\
(1,3)]\end{array}$} \\
\hline & location & \multicolumn{2}{|c|}{ [CL-117] } & \multicolumn{2}{|c|}{$[\mathrm{DE}-308]$} \\
\hline & repetition-period & \multicolumn{2}{|c|}{$\begin{array}{l}02 / 18 / 2013, \\
06 / 21 / 2013]\end{array}$} & \multicolumn{2}{|c|}{$\begin{array}{l}02 / 18 / 2013 \\
06 / 21 / 2013]\end{array}$} \\
\hline & preferred-shorter-free-time & \multicolumn{2}{|l|}{ nil } & \multicolumn{2}{|c|}{$\mathrm{Y}$} \\
\hline \multirow{3}{*}{$\begin{array}{l}\text { Behavior } \\
\text { Attributes }\end{array}$} & attendance-rate & \multicolumn{2}{|l|}{0.90} & \multicolumn{2}{|l|}{0.6} \\
\hline & activity-arrival-trend & \multicolumn{2}{|c|}{$\begin{array}{l}\text { Normal }(-12 \%, 6 \%) \\
\text { Normal }(-12 \%, 6 \%) \\
\text { Normal }(-6 \%, 2 \%)\end{array}$} & \multicolumn{2}{|c|}{$\begin{array}{l}\text { Uniform }(-5 \% \text {, } \\
5 \%)\end{array}$} \\
\hline & activity-leaving -trend & \multicolumn{2}{|c|}{$\begin{array}{l}\text { Normal }(3 \%, 2 \%) \\
\operatorname{Normal}(3 \%, 5 \%)\end{array}$} & \multicolumn{2}{|c|}{$\begin{array}{l}\text { Uniform }(-5 \% \text {, } \\
5 \%)\end{array}$} \\
\hline
\end{tabular}

\section{B. Phase 2 Data Preparation}

The objective of data preparation is to transform the principle-like data collected at phase 1 into concrete data. It is possible to use RFID (Radio Frequency IDentification) technology to track the movement of users [12] and use those tracking data as the data required in this step.

We use activity simulation model to randomly generate 
users' timetables based on the examples in Table I. The Albatross [14] was adapted for data generation in the preparation phase. The simulation deals with the fixed activities before the unfixed activities because most users put the fixed activity on the schedule first, then use the left free time for their unfixed activities. In the study these two parts of activity simulation processes are briefly described as follows:

\section{Fixed Activities Simulation Process}

For each fixed activity, the model first generates activity attributes (i.e., schedule-specs and location) and checks its repetition period, and only deals with the repetition period that falls within the desired simulation time period. Because the schedule-specs. (i.e., activity start time and duration) and location of a fixed activity are deterministic and cannot be changed, the process stops and produce a warning if a conflict is found. Then the model determines the actual values for the behavior attributes, which are probabilistic, to describe how a member participates in this activity, including the attendance-rate, activity-arrival-trend, and activity-leaving-trend. Finally, the model repeats the above process until the simulated time schedule of all fixed activities associated with the member is completed.

\section{Unfixed Activities Simulation Process}

For each unfixed activity, similarly, the model checks its repetition period, and only deals with the repetition period that falls within the desired simulation time period. And the model randomly determines the activity frequency and other activity attributes (i.e., duration and location). If the preferred-shorter-free-time is YES, the process then attempt to find the shortest free time currently available that can still accommodate the activity. Under the constraint set by the user-defined maximum number of trials, the attempt continues until a successful time slot is found. Next, the process generates the behavior attributes of the unfixed activity, which is the same as fixed activity. The loop continues until all multiple occurrences of the unfixed activity have been determined. The final outcome of the simulation process is the activity timetable consisted of all individual time tables.

\section{E. Phase 3 Data Mining}

Based on the activity timetable, the system mines the data by identifying the movement pattern, including movement pattern determination, pattern decomposition, pattern counting, and conditional probability transforming. Readers who are interested in the detail of this phase may refer to [12]. The output of this phase is the values of movement relation between various functions $\left(R_{f i j}\right)$. A function $\mathrm{A}$, with a high movement relation with another function $\mathrm{B}$, indicates that many users went to participate in $\mathrm{B}$ after completing $\mathrm{A}$ function.

\section{F. Phase 4 Optimization}

Phase 4 maximizes the objective function under designated constraints by finding the best assignment of functions to spaces. This research uses the fast messy genetic algorithms (fmGA) to optimize the objective function. The objective function used in this research is based on the concept proposed by Koopmans and Beckman [15]. Equation (1) is the objective function, which is a weighted average of two parts. First, $\left(X_{f i s i} \times P_{f i s i}\right)$ represents the assessment of the suitability of a function assigned to a space. For example, a classroom assigned to a large space is more suitable than to a small space. Secondly, $\left(X_{f i s i} \times X_{f j s j} \times D_{s i s j} \times R_{f i j j}\right)$ represents the assessment of a function assigned to a space from the perspective of the moving distance $\left(X_{f i s i} \times D_{\text {sisj }}\right)$ based on the movement relation $\left(X_{f j s j} \times R_{f i f j}\right)$. For example, strong related functions assigned to neighboring spaces may have a higher assessment value than that to spaces at a distance.

$$
\operatorname{Max} O=W_{1}\left(\frac{\sum_{f_{i}} \sum_{s_{i}} X_{f s_{i}} \times P_{f, s_{i}}}{n}\right)+W_{2}\left(\frac{\sum_{f_{i}} \sum_{s_{i}} \sum_{f_{j}} \sum_{s_{j}} X_{f s_{i}} \times X_{f, s_{j}} \times D_{s, s_{j}} \times R_{f f f_{j}}}{n}\right)
$$

\section{Subjected to}

$$
\begin{aligned}
& X_{f_{j} s_{i}}=0 \text { if } X_{f_{i} s_{i}}=1 \text { and } f_{j} \neq f_{i}, \\
& X_{f_{i} s_{j}}=0 \text { if } X_{f_{i} s_{i}}=1 \text { and } s_{j} \neq s_{i} .
\end{aligned}
$$

where $O=$ objective function; $X_{f i s i}=$ permutation matrix variable (the value is 1 if function $f_{i}$ is assigned to space $s_{i}$, and is 0 if not assigned to $\left.s_{i}\right) ; P_{f i s i}=$ suitability preference of function $f_{i}$ assigned to space $s_{i} ; D_{s i s j}=$ distance between spaces $s_{i}$ and $s_{j} ; R_{f i f j}=$ movement relation of functions $f_{i}$ and $f_{j}$ (the value is between 0 and 1 , where 0 represents no sequential movement pattern exists between functions $f_{i}$ and $f_{j}$, and 1 represents the use of $f_{i}$ always followed by the use of $f_{j}$ ); $n=$ total number of functions; $W_{l}, W_{2}=$ the weights between 0 and 1 .

\section{G. Phase 5 Verification}

The movement simulation module uses the cellular automata (CA) model [16] to simulate occupants' movement trajectories in a building. The CA model is a discrete dynamical system that simulates complex behaviors based on simple computational models. In addition, it requires the modeling of a discrete $W \times W$ cell grid in a two-dimensional area (i.e., the size of the space a user occupies at any point in time), the moving field (i.e., the possible cells that a user can move in each discrete time step during a simulation), and a preference function governing the preferred moving direction of a human. The module allows decision makers to verify the function assignment and demonstrate the users' movement trajectory in 2D and 3D animations. In addition, the module generates a variety of statistics such as the total movement distance for various types of users, usage density of spaces, and cumulative flow density of corridors to assess the service level of a building.

\section{H. Phase 6 Refinement}

There are some service indexes that may be difficult to be represented in the objective function of the optimization phase. For example, one may want to adjust the building layout for qualitative reasons such as the consideration of interference between functions (e.g., classroom should not be adjacent to a professor's office to avoid noise interference), scenic view, safety, and theft concerns. Other reasons may be quantitative, such as those that are difficult to express in an objective function. For example, avoiding movement flow 
congestion may be difficult to express in the objective function because there are theoretically an infinite number of congestion points in continuous corridors. The adjustment for new considerations may also come to mind when decision makers see simulation results.

\section{CASE STUdy}

We conducted an experiment based on a real case to show the research concept and to evaluate the performance of the proposed model. The following sections first introduce the case and then demonstrate the activity simulation and the function assignment results.

\section{A. Case}

The case used in this experiment is the building of the Civil Engineering Department of National Chiao-Tung University in Hsinchu, Taiwan. The building is a 4-story courtyard building with a total floor area of $6,616 \mathrm{~m}^{2}$, as showed in Fig. 2 . The experiment used 32 key spaces (numbered from $s_{1}$ to $\left.s_{32}\right)$ as case study, consisting of an administration office $\left(f_{6}\right)$, a library $\left(f_{1}\right)$, a seminar room $\left(f_{3}\right), 17$ laboratories $\left(f_{8}, f_{9}, f_{15}, f_{16}\right.$, $\left.f_{18}, f_{21}, f_{22}, f_{23}, f_{24}, f_{25}, f_{26}, f_{27}, f_{28}, f_{29}, f_{30}, f_{31} \& f_{32}\right), 3$ meeting rooms $\left(f_{4}, f_{5} \& f_{17}\right)$, and 9 classrooms $\left(f_{2}, f_{7}, f_{10}, f_{11}, f_{12}, f_{13}, f_{14}\right.$, $f_{19} \& f_{20}$ ), situated separately at 4 different floors of the building. The 32 spaces can be divided into 3 groups, i.e., large (L: $90-180 \mathrm{~m}^{2}$ ), medium (M: 45-80 $\mathrm{m}^{2}$ ), and small (S: $15-35 \mathrm{~m}^{2}$ ) spaces. Besides, subjects were 510 undergraduate and graduate students in this experiment and they were divided into 58 groups (the group NO.1 to NO. 58) based on their professional requisite or elective courses.

\section{B. Activity Simulation and Function Assignment Results}

To maintain the uncertain nature of activity participation, the activity simulation module creates an activity timetable for each user based on the probabilistic functions assigned to the user groups where he/she belongs. Fig. 3 shows an example of activity timetable, which is for NO.11 group (Hydraulic Construction Junior). It lists the exact time, location, and activities of the group.

After analyzing the timetables of all groups based on the data mining phase, we can gain the values of movement relation between various functions $\left(R_{f i f j}\right)$. Then function-space assignment is optimized using fmGA based on the Equation (1) so that the total moving distance for the groups to participate in their activities is the minimum.

The fmGA took 250 seconds with the maximum generation equal to 200. As shown in Fig. 4, it actually required only 117 generations converging the optimal assignment $\left(\mathrm{R}_{1}\right)$. The performance is acceptable considering the problem has a combination size of $2.631 \times 10^{35}(=32$ !). In addition, with 117 generations of 448 populations, the number of solutions searched for by the proposed model was $52,416(=448 \times 117)$, which was only $1.992 \times 10^{-31}$ $\left(=52,416 / 2.631 \times 10^{35}\right)$ of the search space.

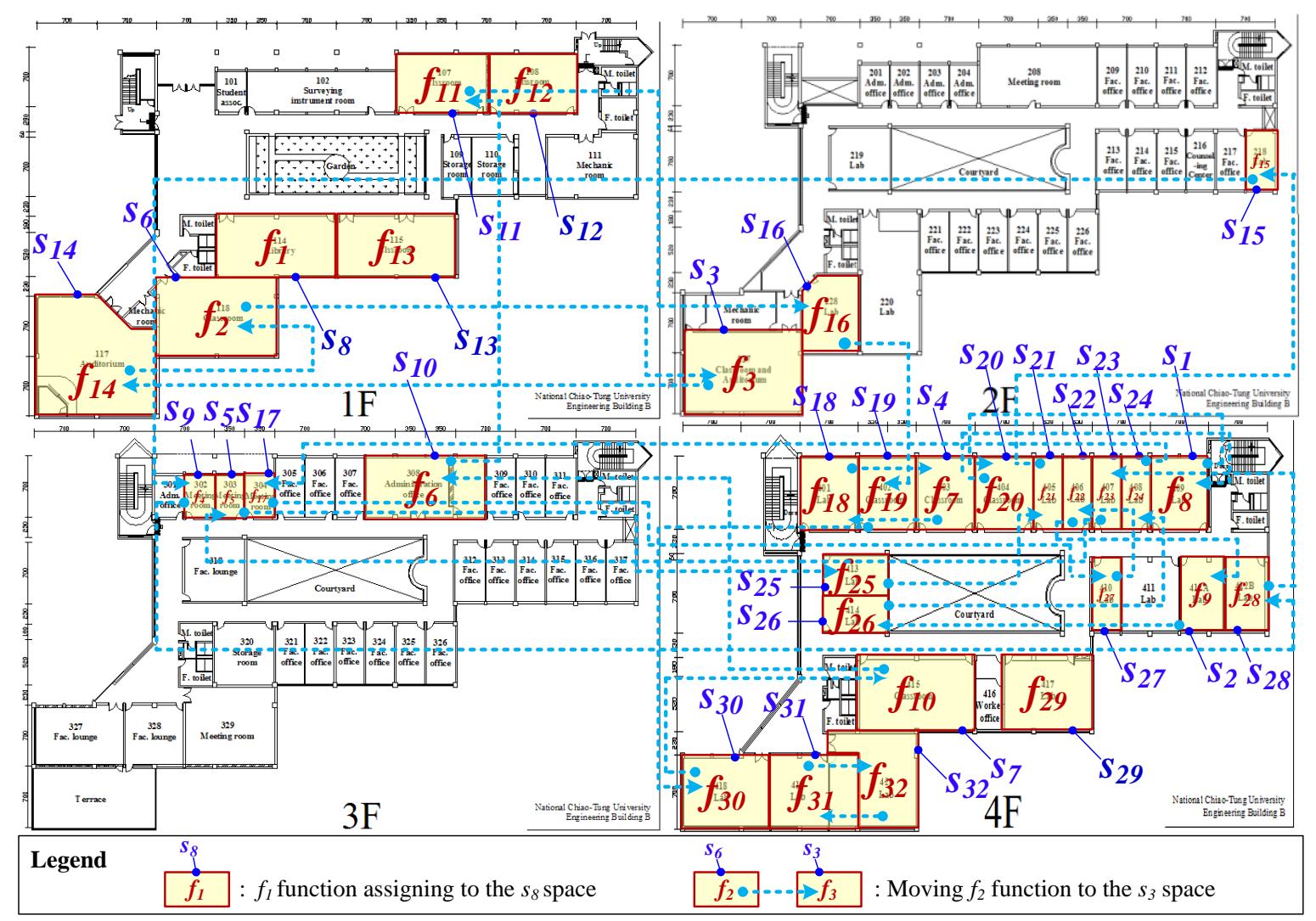

Fig. 2. Comparison of architect's original function assignment and the assignment result $R_{1}$.

The $\mathrm{R}_{1}$ version has a $\left.4.96 \%=(1.0071-0.9595) / 0.9595\right)$ higher objective value than the architect's original version $\left(\mathrm{A}_{0}\right)$. As showed in Fig. 2, the functions are also assigned to the space sizes most-preferred by the administrator (e.g., $f_{3}$ is assigned to the large spaces of $s_{14}, f_{15}$ is assigned to the small spaces of $s_{9}$ ). Additionally, the functions having larger are also placed at least at the same floor (e.g., $f_{6} \& f_{1}, f_{14} \& f_{1}, f_{3} \& f_{1}$, $f_{13} \& f_{1}, f_{12} \& f_{13}, f_{3} \& f_{6}$ are on floor 1$)$. 


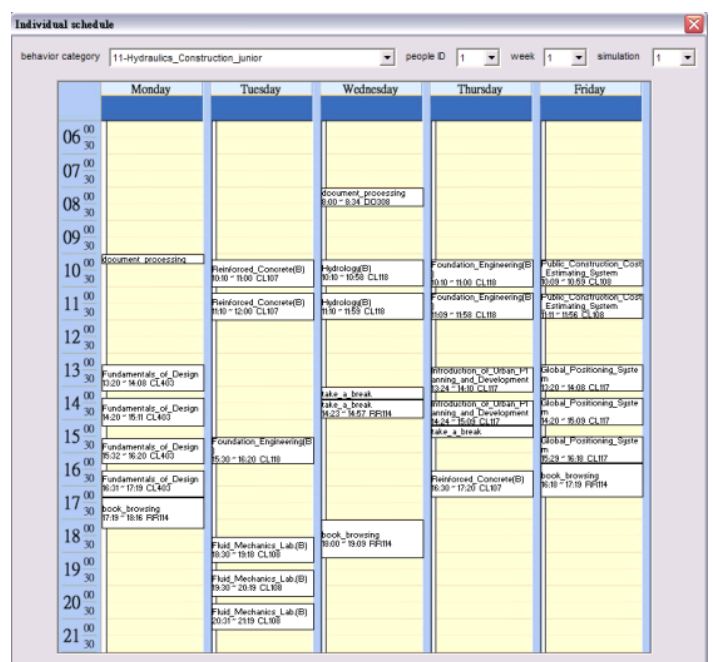

Fig. 3. An activity timetable for NO.11 group.

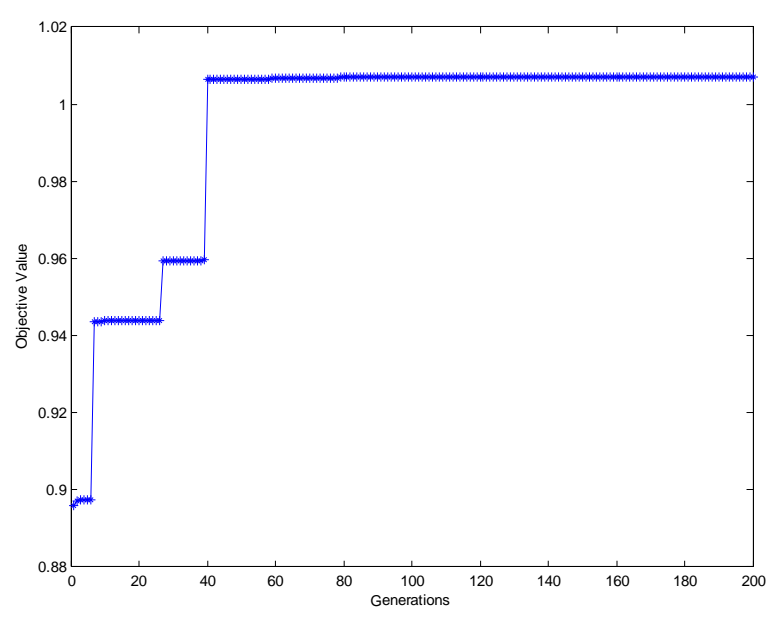

Fig. 4. Objective value of result $R_{1}$.

\section{CONCLUSION}

This research describes the integration of an activity-based simulation model and a space-function assignment optimization model with six phases, including data collection, data preparation, data mining, optimization, verification, and refinement. The Albatross was adapted for data generation in the preparation phase. The fmGA was adopted for the optimization phase. The CA model was also adapted to simulate the movement in the verification phase. The paper also uses a real case to demonstrate the use of the proposed model, and has shown a $4.96 \%$ improvement on the objective value. We expect a greater improvement for a more complex problem where a solution of an experienced architect may have more room for improvement.

\section{ACKNOWLEDGMENT}

This work was partially supported by the National Science Council grants (NSC 99-2221-E-009-133-MY3). Their support is greatly appreciated.

\section{REFERENCES}

[1] C. F. Reinhart, "Lightswitch-2002: A model for manual and automated control of electric lighting and blinds," Solar Energy, vol. 77, pp. 15-28, 2004.

[2] H. Rijal, P. Tuohy, M. Humphreys, J. Nicol, A. Samuel, and J. Clarke, "Using results from field surveys to predict the effect of open windows on thermal comfort and energy use in buildings," Energy and Buildings, vol. 39, pp. 823-836, 2007.
[3] J. A. Clarke, J. Cockroft, S. Conner, J. W. Hand, N. J. Kelly, and R. Moore, "Simulation-Assisted control in building energy Management systems," Energy and Buildings, vol. 33, pp. 933-940, 2002.

[4] A. S. Ramkumar, S. G. Ponnambalam, and N. Jawahar, "A new iterated fast local search heuristic for solving QAP formulation in facility layout design," Robotics and Computer-Integrated Manufacturing, vol. 25, pp. 620-629, 2009.

[5] P. Hoes, J. L. M. Hensen, M. G. L. C. Loomans, B. D. Vries, and D. Bourgeois, "User behavior in whole building simulation," Energy and Buildings, vol. 41, pp. 295-302, 2009.

[6] A. Ekholm and S. Fridqvist, "A concept of space for building classification, product modelling, and design," Automation in Construction, vol. 9, no. 3, pp. 315-328, May 2000.

[7] V. Tabak and B. D. Vries, "Methods for the prediction of intermediate activities by office occupants," Building and Environment, vol. 45, pp. 1366-1372, 2010.

[8] W. Shen, Q. Shen, and Q. Sun, "Building information modeling-based user activity simulation and evaluation method for improving designer-user communications," Automation in Construction, vol. 21, pp. 148-160, 2012.

[9] G. Zimmerman, "Modeling and simulation of individual user behavior for building performance predictions," presented at the 2007 Summer Computer Simulation Conference, 2007.

[10] J. Lertlakkhanakul, J. W. Choi, and M. Y. Kim, "Building data model and simulation platform for spatial interaction management in smart home," Automation in Construction, vol. 17, pp. 948-957, 2008.

[11] H. Y. Lee, I. T. Yang, and Y. C. Lin, "Laying out the occupant flows in public buildings for operating efficiency," Building and Environment, vol. 51, pp. 231-242, 2012

[12] R. J. Dzeng, W. C. Wang, and F. Y. Hsiao, "Function-Space assignment and movement simulation model for building renovation," Journal of Civil Engineering and Management, 2013.

[13] S. Doherty and K. Axhausen, "A conceptual model of the weekly household activity-travel scheduling process," presented at the Network on European Communications and Transport Activities Research Euro-conference, Israel, 1998.

[14] T. Arentze and H. Timmermans "A learning-based transportation oriented simulation system," Transportation Research Part B, vol. 38, pp. 613-633, 2004

[15] T. C. Koopmans and M. Beckman, "Assignment problems and the location of economic activities," Econometrica, vol. 25, no.1, pp. 53-76, Jan. 1957.

[16] H. Yue, H. Hao, X. Chen, and C. Shao, "Simulation of pedestrian flow on square lattice based on cellular automata model," Physica A, vol. 384, pp. 567-588, 2007.

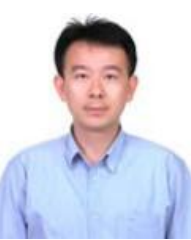

Ren-Jye Dzeng is a professor at the Department of Civil Engineering, National Chiao-Tung University, Taiwan. $\mathrm{He}$ is also acting as the CEO of the Eco-City: Smart Living Technologies Integrated Center, Taiwan. $\mathrm{He}$ received his Ph.D. from the Department of Civil and Environmental Engineering, University of Michigan, Ann Arbor, USA. His research interests include construction engineering \& management, artificial intelligence, RFID, application of brain EEG and virtual reality for construction safety.

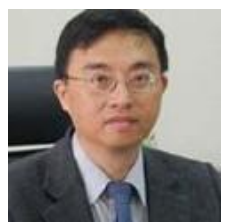

Chin-Teng Lin is a provost/chair professor at the Department of Electrical Engineering and Computer Science, National Chiao-Tung University, Taiwan. He is also acting as the Director of the Eco-City: Smart Living Technologies Integrated Center, Taiwan. He received his Ph.D. from the Department of Electrical Engineering, University of Purdue, West Lafayette, USA. His research interests include Intelligent technology, computational intelligence, fuzzy neural networks (FNN), intelligent transportation systems, robotics and intelligent sensing, intelligent control, multimedia hardware/software, cognitive neuro-engineering, and brain computer interface.

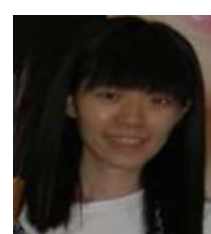

Fan-Yi Hsiao is a Ph.D Candidate at the Department of Civil Engineering, National Chiao-Tung University, Taiwan. She received her Master degree from the Department of Civil Engineering, National Chiao-Tung University, Taiwan. Her research interests include construction engineering \& management, artificial intelligence, and RFID application. 